\title{
DISTRIBUTION OF LRC FOR TESTING SPHERICITY OF A COMPLEX MULTIVARIATE GAUSSIAN MODEL
}

\author{
D. K. NAGAR and S. K. JAIN \\ Departinent of Statistics \\ University of Rajasthan \\ Jaipur, Rajasthan, India \\ and
}

\section{A. K. GUPTA}

Department of Mathematics and Statistics

Bowling Green State University

Bowling Green, Ohio 43403, U.S.A.

(Received February 13, 1984)

ABSTRACT. In this paper, exact null distribution of the likelihood ratio criterion for testing sphericity structure in a complex multivariate normal covariance matrix is obtained in computable series form. The method of inverse Mellin transform and contour integration has been used. Certain special cases are given explicitly in terms of the hypergeometric functions.

KEY WORDS AND PHRASES. Exact distribution, moments, inverse Mellin transform, series representation, Meijer's G-function.

1980 Mathematics Subject Classification Code. $62 H 10$

1. INTRODUCTION.

Let $\underline{z}^{\prime}=\left(\underline{z}_{1}^{\prime}, \ldots, \underline{z}_{q}^{\prime}\right)$ be distributed as a complex multivariate normal with mean vector $\underline{\mu}^{\prime}=\left(\underline{\mu}_{1}^{\prime}, \ldots, \underline{\mu}_{q}^{\prime}\right)$ and Hermitian positive definite covariance matrix $\Sigma$, and let $\underline{z}_{t}$ be of order $p_{t} \times 1, t=1, \ldots, q$. Also, Let $E\left\{\left(\underline{z}_{t}-\underline{\mu}_{t}\right)\left(\underline{z}_{j}-\underline{\mu}_{j}\right){ }^{\prime}\right\}=\Sigma \Sigma_{t j}$, $t, j=1, \ldots, q$. Consider testing the following hypothesis

$$
\mathrm{H}: \Sigma=\left[\begin{array}{ccccc}
\sigma_{1}^{2} \mathrm{I} \mathrm{p}_{1} & 0 & 0 & \ldots & 0 \\
0 & \sigma_{2}^{2} \mathrm{I}_{2} & 0 & \ldots & 0 \\
\cdot & \cdot & . & & \cdot \\
\cdot & \cdot & . & & \cdot \\
\cdot & 0 & . & & \cdot \\
0 & 0 & 0 & \ldots & \sigma_{\mathrm{q}}^{2} \mathrm{I} \mathrm{p}_{\mathrm{q}}
\end{array}\right] \text { vs. A: } \neq \mathrm{H}
$$

where $\sigma_{t}^{2}>0$ is unknown, $I_{p_{t}}$ is the identity matrix of order $p_{t}, t=1, \ldots, q$ and $\mathrm{p}_{1}+\ldots+\mathrm{p}_{\mathrm{q}}=\mathrm{p}$.

It is easy to see that the likelihood ratio criterion for testing $H$ is a one 
to one function of

$$
U=|A| / \prod_{t=1}^{q}\left(\operatorname{tr} A_{t t} / p_{t}\right)^{p_{t}}
$$

where

$$
A=\left(A_{t j}\right) \text { with } A_{t j}=\sum_{k=1}^{N}\left(\underline{z}_{t k}-\underline{z}_{t} \cdot\right)\left(\bar{z}_{j k}-\bar{z}_{j} \cdot\right)^{\prime}, N z_{t} .=\sum_{k=1}^{N} \underline{z}_{t k},
$$

$\underline{z}_{t k}$ is the $k$-th independent observation on $\underline{z}_{t}, k=1, \ldots, N, t=1, \ldots, q$. The $h$-th moment of $U$ is given in Nagarsenker and Nagarsenker [1] as

$$
E\left(U^{h}\right)=\prod_{t=1}^{q}\left\{\frac{p_{t}^{h p_{t}} \Gamma\left(n p_{t}\right)}{\Gamma\left(p_{t}(n+h)\right\}}\right\} \underset{j=1}{p}\left\{\frac{\Gamma\{(n+1-j)+h\}}{\Gamma(n+1-j)}\right\}
$$

where $n=N-1(\geq p)$, and $\operatorname{Re}(h)>-n-1+p$.

Note that when $q=1$, the hypothesis defined in (1.1) is the well known Mauchly's sphericity hypothesis. The non-null distribution of sphericity criterion was given by Pillai and Nagarsenker [2] in series involving zonal polynomials and G-functions. Mathai [3] expanded G-functions in series involving Psi and Zeta functions. Nagarsenker and Das [4] derived the null distribution and gave percentage points. Krishnaiah, Lee and Chang [5] approximated the distribution of certain power of the test criterion by Pearson's type I distribution.

Recently, Nagarsenker and Nagarsenker [1] derived the distribution of $U$ for testing (1.1) in terms of beta and chi-sqaure series. They have also obtained asymptotic results using Box's method.

In this article the exact null distribution of the test statistic $U$ for testing (1.1) is derived in terms of generalized hypergeometric function as well as in series form by using inverse Mellin transform, definition of G-function and calculus of residues. First in section 2 the density is expressed in Meijer's G-function and some special cases are given explicitly. In section 3, this density is given in series of the Psi and Zeta functions. Some special cases for $p \leq 5$ have also been given.

2. THE DENSITY IN MEIJER'S G-FUNCTION.

Using inverse Mellin transform and the expression (1.3) the density of $U$ is given by

$$
f(u)=(2 \pi \omega)^{-1} \int_{C} u^{-1-h} E\left(U^{h}\right) d h, \quad 0<u<1,
$$

where $\omega=(-1)^{\frac{1}{2}}$, and $C$ is the contour enclosing all the poles of the integrand. Simplifying (1.3) by using Gauss-Legendre multiplication formula (Luke [6]), we have

where

$$
E\left(U^{h}\right)=K\left(p_{1}, \ldots, p_{q} ; n\right) \underset{j=1}{p} \Gamma(n-p+j+h) / \prod_{t=1}^{q} \prod_{j=0}^{p_{t}-1} \Gamma\left(n+h+j / p_{t}\right)
$$

$$
K\left(p_{1}, \ldots, p_{q} ; n\right)=\left\{(2 \pi)(p-q) / 2, \prod_{j=1}^{p} \Gamma(n+1-j)\right\} \prod_{t=1}^{q}\left\{\Gamma\left(n p_{t}\right) / p_{t}^{n p_{t}-\frac{1}{2}}\right\} .
$$


Now, substituting (2.2) in (2.1) and subsequently putting $n-p+h=\alpha$, the density is obtained as

$$
\begin{gathered}
f(u)=K\left(p_{1}, \ldots, p_{q} ; n\right)(2 \pi \omega)^{-1} u^{n-p-1} \int_{C_{1}}\left\{\prod_{j=1}^{p} \Gamma(\alpha+j) / \prod_{t=1}^{q} \prod_{j=0}^{p_{t}-1} \Gamma\left(\alpha+p+j / p_{t}\right) u^{-\alpha} d \alpha,\right. \\
0<u<1
\end{gathered}
$$

where $c_{1}$ is the changed contour and the constant $K\left(p_{1}, \ldots, p_{q} ; n\right)$ is defined by $(2.3)$.

Using the definition of G-function (Luke [6]), the density given in (2.4) can be put in the form of the following theorem.

THEOREM 2.1. The p.d.f. of the test statistic $U$ defined by (1.2) for testing the hypothesis (1.1), in the null case, is given by

$$
\begin{aligned}
& f(u)=k\left(p_{1}, \ldots, p_{q} ; n\right) u^{n-p-1} \\
& \text { - } G_{p-1, p-1}^{p-1,0}\left[\left.u\right|_{\{j, j=1, \ldots, p-1\}} ^{\left.\{p, \text { repeated } q-1 \text { times }\},\left\{p+j / p_{t}, j=1, \ldots, p_{t}-1, t=1, \ldots, q\right\}\right]}\right. \text {, } \\
& 0<\mathrm{u}<1
\end{aligned}
$$

where $K\left(p_{1}, \ldots, p_{q} ; n\right)$ is given by $(2.3)$.

When $q=1$, we get the distribution of the sphericity criterion as a corollary of the above theorem.

COROLLARY 2.1. The p.d.f. of the test statistic $U=|A| /(\operatorname{trA} / \mathrm{p})^{p}$ for testing $\mathrm{H}: \quad \Sigma=\sigma^{2} \mathrm{I}_{\mathrm{p}}$ in the complex normal population is given by

$$
f(u)=K(p ; n) u^{n-p-1} G_{p-1, p-1}^{p-1,0}\left[\left.u\right|_{j, j=1, \ldots, p-1} ^{p}+j / p, j=1, \ldots, p-1\right], \quad 0<u<1
$$

where the constant $\mathrm{K}(\mathrm{p} ; \mathrm{n})$ is obtained from $(2.3)$ by substituting $\mathrm{q}=1$ and $\mathrm{p}_{1}=\mathrm{p}$.

The following special cases of (2.5) are stated below by using the results on G-function.

$$
\begin{aligned}
& \text { (i) } q=1, p=2 \\
& f(u)=\{B(n-1,3 / 2)\}^{-1} u^{n-2}(1-u)^{\frac{1}{2}}, \quad 0<u<1
\end{aligned}
$$

where $B(a, b)=\Gamma(a) \Gamma(b) / \Gamma(a+b)$.

(ii) $\mathrm{q}=1, \mathrm{p}=3$

$$
\begin{aligned}
& f(u)=\left\{\pi \Gamma(3 n) / 3^{3 n+\frac{1}{2}} \Gamma(n) \Gamma(n-1) \Gamma(n-2)\right\} u^{n-3} \\
& \quad(1-u){ }_{2}^{3} F_{1}(4 / 3,5 / 3 ; 4 ; 1-u), 0<u<1 .
\end{aligned}
$$

(iii) $\mathrm{q}=2, \mathrm{p}_{1}=\mathrm{p}_{2}=1$

$$
f(u)=(n-1) u^{n-2}, \quad 0<u<1 .
$$

(iv) $\mathrm{q}=2, \mathrm{p}_{1}=1, \mathrm{p}_{2}=2$

$$
\begin{aligned}
& f(u)=\left\{\Gamma(2 n) / 15 \times 2^{2 n-4} \Gamma(n-1) \Gamma(n-2)\right\} u^{n-3} \\
& \quad(1-u){ }_{2}^{5 / 2} F_{1}(3 / 2,1 ; 7 / 2 ; 1-u), 0<u<1 .
\end{aligned}
$$


(v) $q=3, p_{1}=p_{2}=p_{3}=1$

$$
f(u)=(n-1)^{2}(n-2) u^{n-3}(1-u+u \log u), 0<u<1,
$$

where ${ }_{2} F_{1}$ is the Gauss' hypergeometric function.

3. THE DENSITY IN SERIES FORM.

Denoting the gamma products of the integrand in (2.4) by $\Delta(\alpha)$, and after cancelling out the gammas occuring in the numerator with those of denominator, we get (see Gupta and Nagar [7], and Nagar et. al. [8])

$$
\Delta(\alpha)=\frac{\prod_{j=1}^{p-q} \Gamma(\alpha+j)}{\prod_{j=p-q+1}^{p-1}(\alpha+j)^{j-p+q} \prod_{t=1}^{q} \prod_{j=1}^{p_{t}-1} \Gamma\left(\alpha+p+j / p_{t}\right)} .
$$

The poles of $\Delta(\alpha)$ are available by equating to zero each factor of $\prod_{i=1}^{\infty}(\alpha+i) \delta_{i}$, where the exponent $\delta_{i}$ gives the order of the pole at $\alpha=-i$, which can be seen to be

$$
\delta_{i}=\left\{\begin{array}{l}
i, \quad i=1,2, \ldots, p-1 \\
p-q, \quad i=p, p+1, \ldots .
\end{array}\right.
$$

The integrand in (2.4) is $\Delta(\alpha) u^{-\alpha}$ and if $\alpha=-i$ is the pole of order $\delta_{i}$ then the residue $R_{i}$ at $\alpha=-i$ is given by

$$
\begin{aligned}
R_{i} & =\frac{1}{\left(\delta_{i}-1\right) !} \lim _{\alpha \rightarrow-i} \frac{\partial^{\delta_{i}-1}}{\partial \alpha_{i}^{-1}}\left\{(\alpha+i){ }^{\delta_{i}} \Delta(\alpha) u^{-\alpha}\right\} \\
& =\frac{1}{\left(\delta_{i}-1\right) !} \lim _{\alpha \rightarrow-i} \frac{\partial^{\delta_{i}-1}}{\delta_{i}-1}\left\{A_{i} u^{-\alpha}\right\}=\frac{1}{\left(\delta_{i}-1\right) !} \lim _{\alpha \rightarrow-i} u_{r=0}^{-\alpha} \sum_{r}^{\delta_{i}-1}\left({ }_{r}^{\delta_{i}-1}\right)(-\log u) A_{i}^{(r)}
\end{aligned}
$$

where

$$
\begin{aligned}
& A_{i}=(\alpha+i){ }^{\delta} i_{\Delta(\alpha)} \\
& \Gamma^{a} i_{(\alpha+i+1)}^{p-q} \Gamma(\alpha+j) \\
& =\frac{j=a_{i}+1}{\prod_{j \in S}(\alpha+j)^{j-p+q} \prod_{j=1}^{a_{i}^{-1}}(\alpha+j)^{j} \prod_{j=a_{i}}^{i-1}(\alpha+j)^{a_{i}} \prod_{t=1}^{q} \prod_{j=1}^{p_{t}-1} r\left(\alpha+p+j / p_{t}\right)} \text {, }
\end{aligned}
$$

$$
S=\{p-q+1, \ldots, p-1\}-\{i\}
$$

and

$$
a_{i}=\left\{\begin{array}{l}
i, \quad i=1,2, \ldots, p-q \\
p-q, \quad i=p-q+1, \ldots .
\end{array}\right.
$$

Now

$$
A_{i}^{(1)}=\frac{\partial A_{i}}{\partial \alpha}=A_{i}\left(\frac{\partial \log A_{i}}{\partial \alpha}\right)=A_{i} B_{i}
$$


and

$$
A_{i}^{(r)}=\frac{\partial^{r-1}\left(A_{i} B_{i}\right)}{\partial \alpha^{r-1}}=\sum_{m=0}^{r-1}(r-1) A_{i}^{(r-1-m)} B_{i}^{(m)}
$$

where

$$
\begin{aligned}
B_{i}= & \frac{\partial \log A_{i}}{\partial \alpha} \\
= & a_{i} \psi(\alpha+i+1)+\sum_{j=a_{i}+1}^{p-q} \psi(\alpha+j)-\sum_{j \in S}(j-p+q)(\alpha+j)^{-1} \\
& -\sum_{j=1}^{a_{i}-1} j(\alpha+j)^{-1}-a_{i} \sum_{j=a_{i}}^{i-1}(\alpha+j)^{-1}-\sum_{t=1}^{q} \sum_{j=1}^{p_{t}^{-1}} \psi\left(\alpha+p+j / p_{t}\right)
\end{aligned}
$$

and

$$
\begin{aligned}
B_{i}^{(m)}= & \frac{\partial B_{i}}{\partial \alpha^{m}}=\frac{\partial^{m+1} \log A_{i}}{\partial \alpha^{m+1}} \\
= & (-1)^{m+1} m !\left[a_{i} \zeta(m+1, \alpha+i+1)+\sum_{j=a_{i}+1}^{p-q} \zeta(m+1, \alpha+j)+\sum_{j \in S}(j-p+q)(\alpha+j)^{-1-m}\right. \\
& \left.+\sum_{j=1}^{a_{i}-1} j(\alpha+j)^{-1-m}+a_{i} \sum_{j=a_{i}}^{i-1}(\alpha+j)^{-1-m}-\sum_{t=1}^{q} \sum_{j=1}^{p_{t}} \zeta\left(m+1, \alpha+p+j / p_{t}\right)\right] .
\end{aligned}
$$

From (3.3) we can write

$$
R_{i}=\frac{u^{i}}{\left(\delta_{i}-1\right) !} \sum_{r=0}^{\delta_{i}^{-1}}\left({ }_{i}^{\delta_{r}^{-1}}\right)(-10 g u){ }^{\delta_{i}^{-1-r}} A_{i 0}^{(r)}
$$

where

$$
\begin{aligned}
A_{i 0}^{(r)} & =A_{i}^{(r)} \text { at } \alpha=-i \\
& =\sum_{m=0}^{r-1}(r-1) A_{i 0}^{(r-1-m)} B_{i 0}^{(m)}
\end{aligned}
$$

with

$$
\begin{aligned}
A_{i 0} & =A_{i} \text { at } \alpha=-i \\
& =\frac{\prod_{j \in S}(j-i)^{j-p+q} \prod_{j=1}^{a_{i}-1}(j-i)^{j} \prod_{j=a_{i}}^{i-1}(j-i)^{a} \prod_{t=1}^{q} \prod_{j=1}^{p_{t}} \Gamma\left(-i+p+j / p_{t}\right)}{B_{i 0}}=B_{i} \text { at } a=-i
\end{aligned}
$$




$$
\begin{aligned}
= & a_{i} \psi(1)+\sum_{j=a_{i}+1}^{p-q} \psi(j-i)-\sum_{j \in S}(j-p+q)(j-i)^{-1} \\
& -\sum_{j=1}^{a_{i}-1} j(j-i)^{-1}-a_{i} \sum_{j=a_{i}}^{i-1}(j-i)^{-1}-\sum_{t=1}^{q} \sum_{j=1}^{p_{t}^{-1}} \psi\left(-i+p+j / p_{t}\right)
\end{aligned}
$$

and

$$
\begin{aligned}
B_{i 0}^{(m)}= & B_{i}^{(m)} \text { at } \alpha=-i \\
= & (-1)^{m+1} m !\left[a_{i} \zeta(m+1,1)+\sum_{j=a_{i}+1}^{p-q} \zeta(m+1, j-i)+\sum_{j \in S}(j-p+q)(j-i)^{-1-m}\right. \\
& \left.+\sum_{j=1}^{a_{i}-1} j(j-i)^{-1-m}+a_{i} \sum_{j=a_{i}}^{i-1}(j-i)^{-1-m}-\sum_{t=1}^{q} \sum_{j=1}^{p_{t}-1} \zeta\left(m+1,-i+p+j / p_{t}\right)\right] .
\end{aligned}
$$

Note that $\psi(\cdot)$ and $\zeta(\cdot, \cdot)$ respectively are the Psi and Zeta functions (Abramowitz and Stegun [9]). Using residue theorem, the integral in (2.4) is evaluated as a sum of residues and the density of $U$ is given in the following theorem.

THEOREM 3.1. The p.d.f. of the test statistic $U$ defined by (1.2) for testing the hypothesis (1.1) is given by

$$
f(u)=K\left(p_{1}, \ldots, p_{q} ; n\right) u^{n-p-1} \sum_{i=1}^{\infty} \frac{u^{i}}{\left(\delta_{i}-1\right) !} \sum_{r=0}^{\delta_{i}-1}\left({ }_{r}^{\delta_{i}-1}\right)(-10 g u){ }^{\delta_{i}-1-r} A_{i 0}^{(r)}
$$

where $K\left(p_{1}, \ldots, p_{q} ; n\right), \quad \delta_{i}$ and $A_{i 0}^{(r)}$ are given by $(2.3),(3.2)$ and (3.12)-(3.14) respectively.

The cumulative distribution function $F(u)$ of $U$ can be obtained by straight forward integration of (3.15).

When $q=1$, we get the distribution of the sphericity criterion as a corollary of the above theorem.

COROLLARY 3.1. The p.d.f. of the test statistics $U$ for testing $H: \Sigma=\sigma^{2} I_{p}$ in the complex normal population is given by

$$
\begin{gathered}
f(u)=K(p ; n) u^{n-p-1}\left[\sum_{i=1}^{p-1} \frac{u^{i}}{(i-1) !} \sum_{r=0}^{i-1}(i-1)(-1 \circ u)\right)^{i-1-r_{A}}(r) \\
\left.+\sum_{i=p}^{\infty} \frac{u^{i}}{(p-2) !} \sum_{r=0}^{p-2}\left({ }^{p}-2\right)(-10 g u)^{p-2-r_{A}(r)}{ }_{i 0}^{(r)}\right]
\end{gathered}
$$

where $K(p ; n)$ and $A_{i 0}^{(r)}$ are obtained from (2.3) and (3.12)-(3.14) by substituting $\mathrm{q}=1, \mathrm{p}_{1}=\mathrm{p}$.

The following special cases of (3.15) are stated below which have been simplified by using the results on gamma, Psi and Zeta functions.

(i) $q=2, p_{1}=1, p_{2}=3$

$$
\begin{aligned}
f(u)= & K(1,3 ; n) u^{n-5}\left[\frac{u}{2 \Gamma(10 / 3) \Gamma(11 / 3)}-\frac{u^{2}(-\log u+3 \log 3-117 / 20)}{\Gamma(7 / 3) \Gamma(8 / 3)}\right. \\
& \left.-\frac{u^{3}}{4 \Gamma(4 / 3) \Gamma(5 / 3)}(-\log u)^{2}+(-2 \log u)(3 \log 3-2)-\pi^{2}+\frac{27}{2}+(3 \log 3-2)^{2}\right\}
\end{aligned}
$$


(ii) $q=2, p_{1}=p_{2}=2$

$$
\begin{aligned}
& +\frac{u^{4}}{\Gamma(1 / 3) \Gamma(2 / 3)} \sum_{j=0}^{\infty} \frac{(2 / 3){ }_{j}(1 / 3) j^{u^{j}}}{(j+1)(j+3)\{(j+2) !\}^{2}}\left\{2 \psi(j+3)+(j+1)^{-1}+(j+3)^{-1}\right. \\
& -\log u-\psi(j+2 / 3)-\psi(j+1 / 3)\}], \quad 0<u<1 .
\end{aligned}
$$

$$
\begin{aligned}
f(u)= & \kappa(2,2 ; n) u^{n-5}\left[\frac{u}{2 \Gamma^{2}(7 / 2)}-\frac{u^{2}(-10 g u+4 \log 2-16 / 3)}{2 \Gamma^{2}(5 / 2)}\right. \\
& -\frac{u^{3}}{4 \Gamma^{2}(3 / 2)}\left\{(-\log u)^{2}+(-2 \log u)(4 \log 2+3 / 2)+41 / 4-\frac{2 \pi^{2}}{3}\right. \\
& \left.+(4 \log 2+3 / 2)^{2}\right\}+\sum_{i=4}^{\infty} \frac{u^{i} \Gamma^{2}\left(i-4+\frac{1}{2}\right)}{(i-3)(i-1)((i-2) !\}^{2} \Gamma^{4}\left(\frac{1}{2}\right)} \\
& \left.\left\{-\log u+2 \psi(i-1)+(i-3)^{-1}+(i-1)^{-1}-2 \psi\left(i-4+\frac{1}{2}\right)\right\}\right], \quad 0<u<1 . \\
\text { (iii) } q=3, & p_{1}=p_{2}=1, p_{3}=2
\end{aligned}
$$$$
f(u)=K(1,1,2 ; n) u^{n-5}\left[\frac{u}{4 \Gamma(7 / 2)}-\frac{u^{2}(-\log u+2 \log 2-11 / 3)}{\Gamma(5 / 2)}\right.
$$$$
-\frac{u^{3}}{4 \Gamma(3 / 2)}\left\{(-\log u)^{2}+(-\log u)\left(2 \log 2+\frac{1}{2}\right)-\frac{\pi^{2}}{3}+\frac{25}{4}+\left(2 \log 2+\frac{1}{2}\right)^{2}\right\}
$$$$
\left.+\sum_{i=4}^{\infty} \frac{\Gamma\left(i-4+\frac{1}{2}\right) u^{i}}{(i-2)(i-3)^{2}(i-1) ! \Gamma^{2}\left(\frac{1}{2}\right)}\right], \quad 0<u<1 \text {. }
$$

(iv) $q=4, p_{1}=p_{2}=p_{3}=p_{4}=1$

$$
f(u)=\frac{(n-1)^{3}(n-2)^{2}(n-3)}{8}\left[1+8 u(2+\log u)-2 u^{2}\left\{(-\log u+5 / 2)^{2}+9 / 4\right\}\right],
$$

(v) $p=5, q=4, p_{1}=2, \quad p_{2}=p_{3}=p_{4}=1$

$$
\begin{aligned}
f(u)= & k(2,1,1,1 ; n) u^{n-6}\left[\frac{u}{108 \Gamma(9 / 2)}+\left(-10 g u+2 \log 2-\frac{167}{30}\right)\left(\frac{-u^{2}}{8 \Gamma(7 / 2)}\right)\right. \\
& +\left\{(-\log u)^{2}+(-2 \log u)\left(2 \log 2-\frac{19}{6}\right)+\frac{259}{36}-\frac{\pi^{2}}{3}+\left(2 \log 2-\frac{167}{6}\right)^{2}\right\} \\
& \left(\frac{-u^{3}}{\Gamma(5 / 2)}\right)+\left\{(-\log u)^{3}+3(-\log u)^{2}\left(2 \log 2+\frac{7}{3}\right)+3(-10 g u)\left(\frac{137}{18}-\frac{\pi^{2}}{3}\right.\right. \\
& \left.\left.+\left(2 \log 2+\frac{7}{3}\right)^{2}\right)+12 \zeta(3)+\frac{355}{54}+3\left(\frac{137}{18}-\frac{\pi^{2}}{3}\right)\left(2 \log 2+\frac{7}{3}\right)+\left(2 \log 2+\frac{7}{3}\right)^{3}\right\} \\
& \left.\left(\frac{u^{4}}{12 \Gamma(3 / 2)}\right)+\frac{u^{5}}{\Gamma\left(\frac{1}{2}\right)} \sum_{j=0}^{\infty} \frac{\left(\frac{1}{2}\right) u^{j}}{(j+3)(j+2)^{2}(j+1)^{3}(j+4) !}\right], 0<u<1 .
\end{aligned}
$$

(vi) $\mathrm{p}=5, \mathrm{p}_{1}=\ldots=\mathrm{p}_{5}=1$ 


$$
\begin{aligned}
f(u)= & (n-4)(n-3)^{2}(n-2)^{3}(n-1)^{4} u^{n-6}\left[\frac{u}{648}-\frac{u^{2}}{16}(-\log u-4)\right. \\
& -\frac{u^{3}}{4}\left\{(-\log u)^{2}-3(-\log u)+\frac{21}{4}\right\} \\
& \left.+\frac{u^{4}}{36}\left\{(-\log u)^{3}+13(-\log u)^{2}+\frac{403}{6}(-\log u)+\frac{1214}{9}\right\}\right], \quad 0<u<1 .
\end{aligned}
$$

\section{REFERENCES}

1. NAGARSENKER, B. N. and NAGARSENKER, P. B. Distribution of the likelihood ratio statistic for testing sphericity structure for a complex normal covariance matrix, Sankhya, B43 (1981), 352-359.

2. PILLAI, K. C. S. and NAGARSENKER, B. N. On the distribution of sphericity test criterion in classical and complex normal populations having unknown covariance matrices, Ann. Math. Statist., 42 (1971), 764-767.

3. MATHAI, A. M. A few remarks about some recent articles on the exact distribution of multivariate test criteria: I, Ann. Inst. Statist. Math., 25 (1973), 566-577.

4. NAGARSENKER, B. N. and DAS, M. M. Exact distribution of sphericity criterion in the complex case and its percentage points, Comm. Statist., 4 (1975), 363-375.

5. KRISHNAIAH, P. R., LEE, J. C. and CHANG, T. C. The distribution of the likelihood ratio statistics for tests of certain covariance structures of complex multivariate normal populations, Biometrika, 63 (1976), 513-519.

6. LUKE, Y. L. The Special Functions and Their Approximations, Vol. I, Academic Press, New York, 1969.

7. GUPTA, A. K. and NAGAR, D. K. Distribution of LR statistic for testing $\mathrm{H}: \underline{\mu}=\underline{\mu}_{0} ; \quad \Sigma=\sigma^{2} \mathrm{I}$ in multivariate complex Gaussian distribution, Department of Mathematics and Statistics, Bowling Green State University, T.R. No. 83-03, 1983.

8. NAGAR, D. K., JAIN, S. K. and GUPTA, A. K. Distribution of LRC for testing sphericity of a complex multivariate Gaussian model, Department of Mathematics and Statistics, Bowling Green State University, T.R. No. 83-09, 1983.

9. ABRAMOWITZ, M. and STEGUN, I.A. Handbook of Mathematical Functions, Dover publications, New York, 1965. 


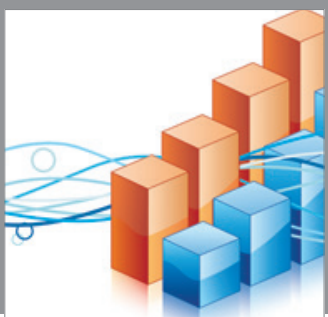

Advances in

Operations Research

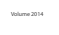

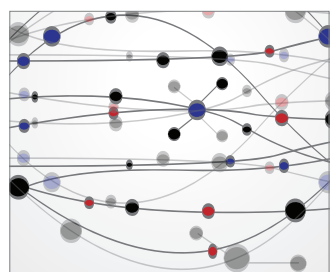

\section{The Scientific} World Journal
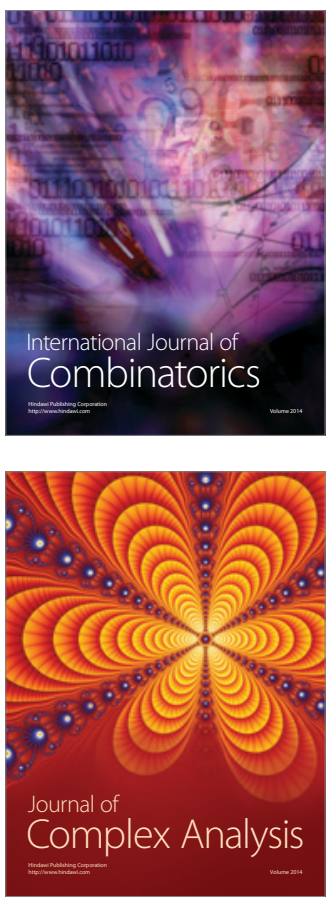

International Journal of

Mathematics and

Mathematical

Sciences
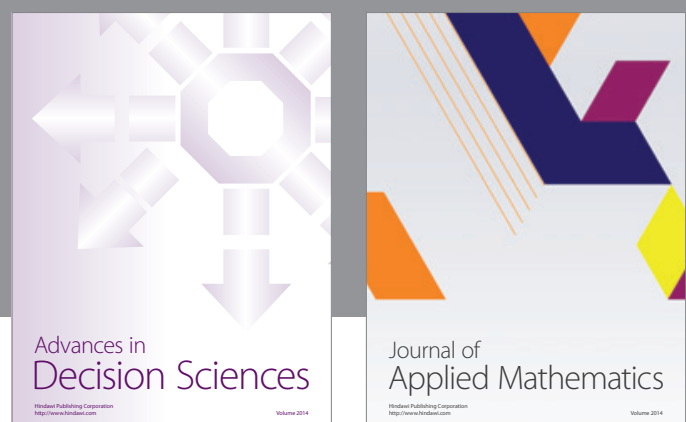

Journal of

Applied Mathematics
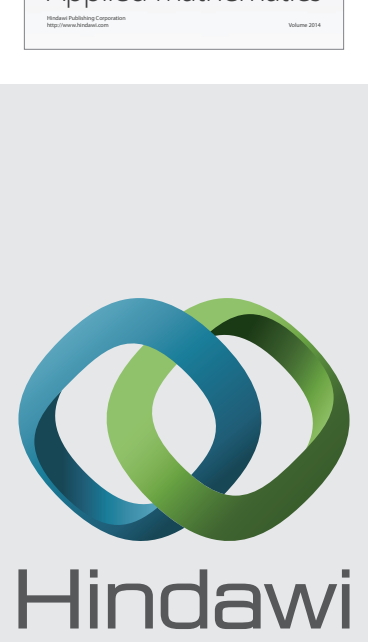

Submit your manuscripts at http://www.hindawi.com
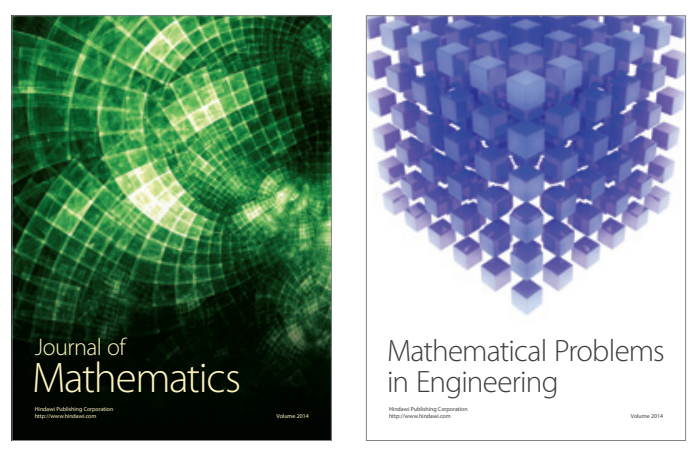

Mathematical Problems in Engineering
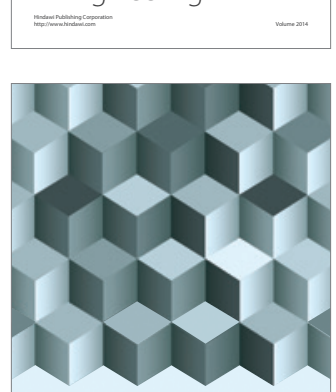

Journal of

Function Spaces
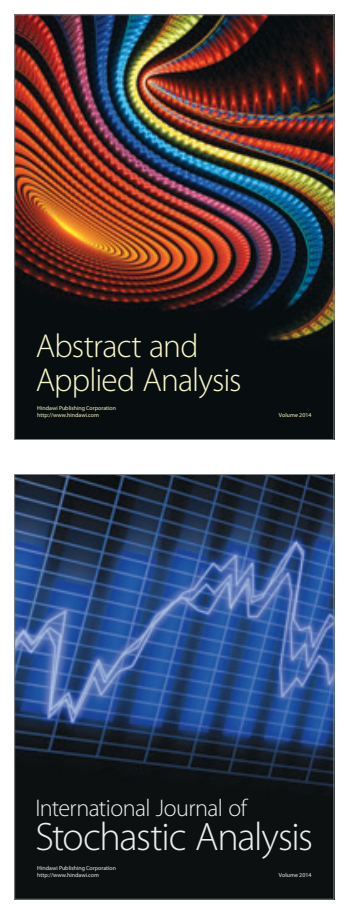

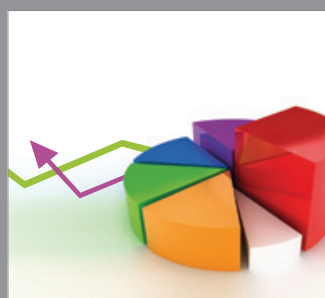

ournal of

Probability and Statistics

Promensencen
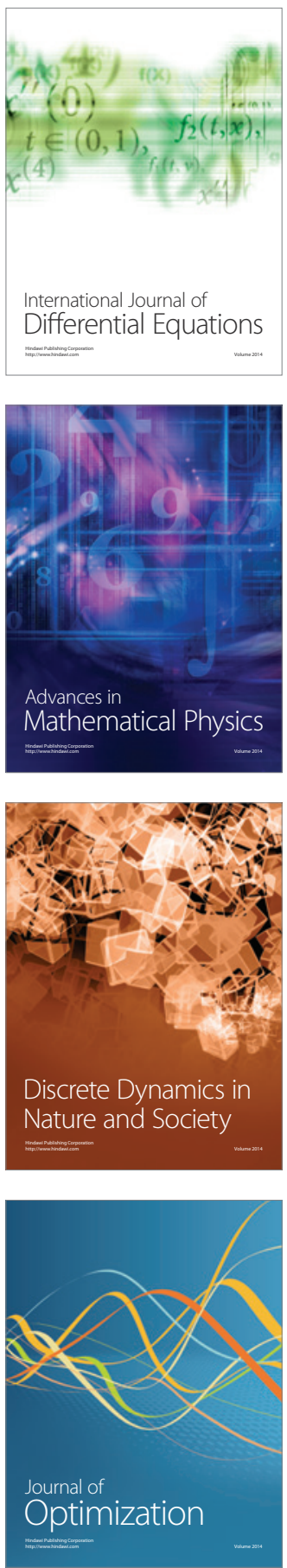